\title{
Fechamento Percutâneo da Comunicação Interatrial
}

\author{
Valmir Fernandes Fontes, Carlos Augusto Cardoso Pedra
}

São Paulo, SP

A comunicação interatrial como lesão isolada representa $7 \%$ de todas as anomalias congênitas do coração e tem grande predomínio no sexo feminino, cerca de dois terços. Sendo um defeito situado ao longo do septo interatrial é fácil compreender que sua localização é variável. Consideramse quatro tipos anatômicos principais: 1) o tipo mais freqüente, o ostium secundum ou da fossa oval, corresponde a $75 \%$ dos casos, podendo ser um defeito único, múltiplo ou apresentar-se com fossa oval multifenestrada; 2) o tipo ostium primum, situado na parte mais baixa do septo, correspondendo a $15 \%$ dos casos; 3 ) o tiposinus venosus, situado na parte mais alta e posterior do septo, tendo como característica a presença de uma ou duas veias pulmonares direitas, abrindo-se anormalmente ao nível da desembocadura da veia cava superior e correspondendo a 8-9\% dos casos; 4) por fim o mais raro, denominado de seio coronariano, usualmente encontrado com anomalia de conexão de uma veia cava superior esquerda persistente, abrindo-se no teto do átrio esquerdo e correspondendo a 1- $2 \%$ dos casos.

O único tipo anatômico que se presta para fechamento percutâneo é o ostium secundum. Sua história natural é bem conhecida. Craig e Selzer ${ }^{1}$ estudaram consecutivamente 128 portadores desse defeito, todos adultos, variando de 18 a 60 anos. O estudo hemodinâmico revelou $22 \%$ dos casos com hipertensão pulmonar, em $15 \%$ com alta resistência vascular pulmonar, achado este mais frequiente na faixa etária dos 20 aos 40 anos. A insuficiência cardíaca foi mais comum nos pacientes idosos, assim como a ocorrência de arritmias crônicas. Campbell ${ }^{2}$ publicou, em 1970, seu estudo sobre a história natural em um grupo selecionado de doentes, todos sintomáticos e portadores de grandes defeitos e verificou que a sobrevida atuarial aos 60 anos foi de apenas $15 \%$ para os portadores de comunicação interatrial, contra $85 \%$ para a população geral, fato inequívoco de que o defeito septal

Instituto Dante Pazzanese de Cardiologia

Correspondência: Valmir Fernandes Fontes - Instituto Dante Pazzanese de Cardiologia - Av. Dr. Dante Pazzanese, 500 - 12 ${ }^{\circ}$ - 04012-180 - São Paulo, SP e-mail: vffontes@uol.com.br

Recebido para publicação em 16/5/01

Aceito em 11/7/01 não é tão inocente como aprioristicamente se pensa. O tratamento da comunicação interatrial sempre foi cirúrgico e vem sendo praticado desde 1951, independente do tipo anatômico. A cirurgia é uma excelente opção, abrange todas as variedades anatômicas, oferece baixo índice de complicações e a mortalidade é próxima de $0 \%$. Apesar destas vantagens, o procedimento é cirúrgico, requer toracotomia, circulação extracorpórea, passagem pela UTI, causa trauma físico e psíquico, etc.

Em casos selecionados, os cirurgiões têm a opção da técnica minimamente invasiva, a incisão cirúrgica é subxifoideana, pequena, menos traumática, contudo o procedimento continua sendo cirúrgico.

O fechamento percutâneo da comunicação interatrial exige o emprego de uma prótese, o tratamento é alternativo e deve oferecer resultados equivalentes ao do tratamento cirúrgico.

Recapitulando, a história, o primeiro dispositivo para fechamento percutâneo da comunicação interatrial foi descrito por King e Mills ${ }^{3}$ em 1974, constava de um par de umbrellas cobertas de dacron com uma armação de aço inoxidável. O sistema liberador era calibroso, com 23Fr de diâmetro. Os autores publicaram, em 1976, experiência inicial de 10 casos, com sucesso em cinco. Devido às dificuldades técnicas de implante e ser um dispositivo ainda primitivo, não teve aceitação pelos intervencionistas da época e o projeto foi então abandonado.

Rashkind após ter padronizado um dispositivo de dupla umbrella para fechamento de canal arterial persistente (PDA umbrella), passou a trabalhar em um projeto para oclusão de comunicação interatrial. Recebeu aprovação do Federal Drug Administration (FDA), dando início em $1987^{4}$. A prótese consistia em uma única umbrella auto-expansível com seis braços articulados de aço inoxidável, recoberta por espuma de poliuretano. Três dos braços apresentavam minúsculos ganchos de fixação a fim de penetrarem nas bordas do defeito fazendo a ancoragem da prótese. A presença dos ganchos de fixação exigia um sistema liberador de grande diâmetro. A experiência clínica preliminar consistiu de 20 casos, obtendo-se sucesso em 13. Hellenbrand e Mullins ${ }^{5}$, Beekman e cols. ${ }^{6}$ também relataram seus primeiros casos. 
A umbrella de Rashkind foi modificada por Lock para dupla umbrella, de formato quadrado, a armação feita de aço inoxidável com quatro hastes articuladas, recobertas de dracon. O sistema liberador foi reduzido para $11 \mathrm{Fr}$, o dispositivo passou a ser denominado de Bard Clamshell Septal Umbrella. Lock e cols. ${ }^{7,8}$, Rome e cols. ${ }^{9}$ publicaram os resultados iniciais.

Em 1990, cerca de 100 portadores de comunicação interatrial haviam sido submetidos a fechamento com a Chamshell, sob aprovação do FDA ${ }^{10}$. O sucesso de implantes foi de $93 \%$, ocorreu embolização em três casos, em dois houve necessidade de resgate cirúrgico. A avaliação tardia demonstrada pelo color Doppler mostrou oclusão completa em $84 \%$ dos casos, revelando alta incidência de fratura das hastes, teve aceitação limitada e nunca recebeu aprovação do FDA para uso rotineiro nos Estados Unidos.

A umbrella oclusora Chamshell foi modificada pela engenharia da Nitinol Medical Technologies Inc. supervisionada por um grupo seleto de cardiologistas intervencionistas, destacando-se os Drs. Mullins, Helebrand, Lock, Benson, entre outros.

As modificações incluíram novo desenho, o aço inoxidável foi substituído pelo nitinol, uma liga metálica super elástica, as hastes foram biarticuladas com a idéia de evitar fraturas, a superfície da prótese foi recoberta por poliéster. O dispositivo passou a ser conhecido com nome de CardioSeal.

Novos estudos clínicos começaram no final de 1996, nos Estados Unidos, Canadá e Europa e o seguimento dos doentes veio mostrar incidência relativamente elevada de shunt residual e fratura de hastes.

O dispositivo inicial CardioSeal sofreu aperfeiçoamento, a estrutura básica foi mantida porém introduziu-se um mecanismo flexível de autocentralização e a prótese é encontrada no mercado em quatro tamanhos: 23, 28, 33 e 40, fechando comunicação interatrial até $20 \mathrm{~mm}$ de diâmetro. A prótese passou a ser conhecida com o nome CardioSeal/Starflex.

Um estudo multicêntrico europeu de outubro/96 a abril/ 99 envolveu 334 pacientes ${ }^{11}$. A taxa de embolização da prótese foi de $4 \%$, a presença de shunt residual ao completar o primeiro ano foi de 20,5\% e fratura de hastes ocorreu em 6,1\%.

O Hospital for Sick Children, Toronto, Canadá, publicou sua experiência de dezembro/96 a julho/98, envolvendo 50 pacientes ${ }^{12}$. Todos tiveram sucesso no implante, embora em $8 \%$ dos casos a prótese tenha sido removida e um segundo dispositivo tenha sido implantado devido ao mau posicionamento inicial. Em um seguimento de 9,9 $\pm 3,2$ meses, identificou-se shunt residual em $46 \%$, medindo menos de $2 \mathrm{~mm}$ em $26 \%$ dos casos, fratura de hastes $14 \%$, prolapso de uma das hastes através do defeito em $32 \%$.

Em 1990 Sideris e cols. ${ }^{13}$ desenvolveram um dispositivo abotoável (button device) composto de três partes: a oclusora, contraoclusora e o sistema de liberação. O oclusor é feito de poliuretano e o esqueleto da prótese é de aço inoxidável revestido de teflon, o contraoclusor tem forma romboidal, também revestido de poliuretano, e a armação de aço coberto de teflon. Uma peça de borracha é suturada no cen- tro da prótese e torna-se abotoável. O dispositivo é liberado com uma bainha $8 \mathrm{Fr}$.

Até os dias de hoje, a prótese recebeu várias modificações, está na quarta geração, tem problemas como dificuldades de abotoamento, embolizações, shunts residuais, etc. Godar e cols. ${ }^{14}$ relataram uma experiência de 1992 a 1997, a prótese foi usada em 73 pacientes consecutivos, o sucesso de implante ocorreu em $75 \%$ dos casos, três pacientes necessitaram de cirurgia imediata, um por embolização e dois devido à presença de grande shunt residual e cavalgamento do dispositivo. Durante o seguimento, mais sete pacientes necessitaram de cirurgia devido à perfuração atrial eshunt residual significativo, tardiamente a oclusão completa ocorreu em $69 \%$ dos casos.

Rao e cols. ${ }^{15}$ apresentaram uma experiência internacional de 200 casos, o índice de sucesso foi de $87 \%$ e a oclusão completa foi de 79\%, 43 meses após o implante.

Devido a resultados pouco satisfatórios, a prótese tem, no momento, pouca aceitação.

Em 1991, Babic ${ }^{16}$ descreveu sua prótese ASDOS, composta de 2 umbrellas contrapostas e auto-expansíveis, com armação de fio de nitinol e revestida de poliuretano, necessitando de um introdutor de liberação de $10 \mathrm{Fr}$. A experiência inicial de 20 casos mostrou sucesso de implante em $80 \%$, elevado índice de complicações e a oclusão completa se deram em apenas $60 \%$ dos casos. Não tem tido aceitação pelos intervencionistas.

A AGA Medical Corporation (USA), desenvolveu uma prótese para oclusão de comunicação interatrial supervisionada pelo Dr. Amplatz, a qual veio se chamar de prótese de Amplatzer. O dispositivo é composto de dois discos oclusores contrapostos, auto-expansíveis, unidos por um núcleo central denominado de stent ou cintura. Os discos são constituídos de uma rede metálica de nitinol, internamente preenchidos por retalhos de poliéster, com a finalidade de aumentar o poder trombótico. A prótese é autocentrável, o disco distal ou do átrio esquerdo é maior 16mm que a cintura e o disco proximal ou do átrio direito é maior $10 \mathrm{~mm}$. Nos dias de hoje encontra-se à disposição dos intervencionistas, próteses de 4 a 38mm de diâmetro.

Sua liberaçãoé feita de maneira simples por meio de uma bainha longa de 6 a 12 Fr de diâmetro e de um cabo liberador de aço inoxidável terminado em forma de parafuso, o qual é parafusado num pino em forma de rosca localizado no centro do disco atrial direito. A rotação horária prende o cabo liberadorà prótese e a rotação anti-horária faz a liberação do dispositivo.

Os trabalhos experimentais em animais foram feitos em suínos, publicados por Sharafuddin e cols. ${ }^{17}$. O emprego na espécie humana teve um crescimento fantástico, é a prótese de maior uso em todo o mundo. O segredo da sua aceitação deve-se as suas grandes vantagens, entre elas: 1) é auto-expansível e autocentrável; 2) é resgatável; 3 ) não estando bem posicionada pode ser recolhida para a bainha e reposicionada tantas vezes seja necessário; 4) é fácil de ser implantada; 5) sua endotelização se faz em seis meses; 6) tem elevado índice de sucesso de implante, de oclusão completa e baixíssimo índice de complicações; 7) alta hospitalar de rotina em $24 \mathrm{~h}$, podendo ser um procedimento ambu- 
latorial; 8) em casos selecionados pode ser implantada tendo como guia único o ecotransesográfico.

Os primeiros trabalhos começaram a ser conhecidos na literatura a partir de $1998{ }^{18}$. Wilkinson e cols. ${ }^{19}$ publicaram sua experiência de 27 pacientes onde obtiveram sucesso em 26 , ou seja, $96 \%$; Thanapouls e cols. ${ }^{20}$ tiveram resultados preliminares equivalentes; Fontes e cols. ${ }^{21}$ publicaram sua experiência inicial de sete casos com $100 \%$ de sucesso de implante. A experiência da nossa Instituição alcança 68 casos, o implante foi completado em 66-97\%, em um dos doentes havia deficiência de bordas na região pósteroinferior, a prótese não adquiriu estabilidade e o procedimento foi abortado. Em outro paciente a valva de Eustáquio era muito proeminente e, na manobra de liberação da prótese, $\mathrm{o}$ tecido de valva se enrroscou no cateter liberador, travando sua liberação. A prótese foi recolhida para a bainha e o procedimento foi abortado. A presença de shunt residual nos dois primeiros anos pós-implante foi verificada em quatro casos, em dois pacientes menor de $2 \mathrm{~mm}$ e em dois entre $2 \mathrm{e}$ $4 \mathrm{~mm}$, sem repercussão clínica e inclusive com normalização do diâmetro do ventrículo direito à luz da ecocardiografia.

Berger e cols. ${ }^{22}$ fizeram um estudo comparativo de resultados e complicações entre o fechamento percutâneo e o fechamento cirúrgico, envolvendo 61 pacientes para cada grupo. A taxa de oclusão completa nos dois grupos foi de 98\%, uma prótese embolizou, embora tenha sido resgatada na sala de hemodinâmica, necessitou de cirurgia; do grupo operado, um paciente teve úlcera duodenal perfurada e outro devido a infecção da toracotomia lateral necessitou de cirurgia plástica, dois pacientes fizeram fibrilação atrial e dois derrame pericárdico. O tempo médio de hospitalização foi de três dias para a prótese e de oito dias para o procedimento cirúrgico. Concluem que as taxas de oclusão e complicações são idênticas, porém com maior morbidade e maior permanência hospitalar, no grupo cirúrgico.

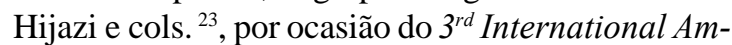
platzer Symposium realizado em Strassbourg, em 2000, apresentaram o Estudo Multicêntrico Internacional, envolvendo 4.008 pacientes. A taxa de implante completado foi de $96,7 \%$, o sucesso clínico definido como oclusão completa ou presença de shunts residuais triviais ou discretos, foi de $99,1 \%$. Em $0,9 \%$ a magnitude de shunts foi de grau médio em $0,7 \%$ e grande $0,2 \%$.

Recentemente, nova prótese chegou ao mercado, denomina-se de Helix Septal Occluder, fabricada pela Gore (W.L. Gore \& Associates, Inc.) a qual é composta de um fio de nitinol com diâmetro de 0,012 pol, coberto por uma membrana de politetrafluoretileno. Na configuração final do seu corpo, o dispositivo forma dois discos flexíveis e arredondados, sua liberação é feita por um sistema liberador $9 \mathrm{Fr}$.

A prótese foi testada em animais de experimentação ${ }^{24}$, os implantes humanos têm sido realizados em Glasgow, na Escócia e em Frankfurt, na Alemanha. A experiência inicial consta de 28 pacientes mostrando o sucesso de implante em 26 casos; um mês após o implante, estudos com color Doppler mostraram oclusão completa em 93\% e 7\% com shunts residuais pequenos. Na nossa Instituição, testamos esta prótese em 6 pacientes: o diâmetro estirado dos defeitos variou de 10 a $20 \mathrm{~mm}$. Um paciente possuía dois defeitos, recebendo duas próteses. Em um seguimento de apenas um mês, um paciente ficou com um discreto "shunt" residual, exatamente o que se apresenta com dois orifícios.

O fechamento percutâneo da comunicação interatrial é consenso mundial? Temos no mercado uma prótese ideal?

A prótese ideal precisa preencher vários requisitos, entre eles: 1) baixo perfil; 2) auto-expansível; 3) autocentrável; 4) técnica de implante simples e facilmente reproduzível; 5) sistema de liberação de pequeno diâmetro; 6) facilidade de ajuste e de resgate antes de ser liberada; 7) durabilidade até completar a endotelização; 8) preservação do fluxo e da função cardíaca; 9) baixo índice de complicações; 10) resultados efetivos comparáveis ao tratamento cirúrgico.

No momento, duas próteses se destacam no cenário internacional para fechamento percutâneo da comunicação interatrial, a de Amplatzer e a CardioSeal/Starflex .

Odispositivo de Amplatzer acumula uma experiência de milhares de casos, tem elevado índice de sucesso e baixíssimo índice de complicações, é oferecida no mercado com diâmetros de 4 a $38 \mathrm{~mm}$. Embora preencha a maior parte dos requisitos de uma prótese ideal, é volumosa, nãoé de baixo perfil, pode ocupar $30 \%$ da cavidade atrial queéum fator limitante para grandes defeitos em crianças com peso abaixo de $15 \mathrm{~kg}$. Outro inconveniente é que o corpo ou cintura corresponde ao diâmetro estirado do defeito septal; é esta estrutura que oclui o defeito e nos casos de comunicação interatrial da fossa oval multifenestrada ou aneurisma da fossa oval com pequena comunicação interatrial, a cintura não se expande dentro de um orifício pequeno, provocando deformações.

Outro ponto a ser levantado é o metal utilizado na confecção da prótese, que é uma liga de níquel e titânio, chamada de nitinol, material utilizado hoje em dia em todas as próteses. O poder tóxico do níquel vem sendo estudado e ainda não é bem conhecido.

A segunda prótese mais utilizada é a CardioSeal/ Starflex, de baixo perfil; ao contrário da Amplatzer, é bem indicada em aneurisma da fossa oval ou quando esta é multifenestrada, contudo emprega-se para defeitos de até $20 \mathrm{~mm}$ de diâmetro estirado, tem elevado índice de fratura de hastes e de shunts residuais.

A prótese de Helix, recém lançada no mercado, é de baixo perfil, usa também o nitinol como metal de armação da sua estrutura, a experiência é pequena e encontra-se em fase de investigação.

O fechamento percutâneo da comunicação interatrial é uma realidade palpável, a prótese ideal está próxima de ser conseguida, a possibilidade de utilizar a de Amplatzer na maioria dos casos e selecionar a Cardioseal/Starflex ou quem sabe a Helix para casos de aneurisma da fossa oval, fossa oval multifenestrada, ou pequenas comunicações interatriais em crianças de baixo peso, parece ser uma conduta adequada.

Não podemos esquecer que o tratamento cirúrgico vem da década de 50 e tem sua história natural bem conhecida. Precisamos também aguardar o seguimento das próteses e escrever sua história natural. 


\section{Referências}

1. Craig RJ, Selzer A. Natural history and Prognosis of atrial septal defect. Circulation 1968; 37: 805-15.

2. Campbell M. Natural history of atrial septal defect. Br Heart J 1970; 32: 820-6.

3. King TD, Thompson SL, Steiner C, Mills NL. Secundum atrial septal defect. Nonoperative closure during cardiac catheterization. Jama 1976; 23: 2506-9.

4. Rashkind WJ, Cuaso CC. Transcatheter closure of atrial septal defects in children, abstracted. Eur J Cardiol 1987; 8: 119-20.

5. Hellembrand WE, Mullins CE. Catheter closure of congenital defects. Cardiol Clin 1989; 7: 351-68.

6. Beekman RH, Rocchini AP, Snider AR, Rosenthal A. Transcatheter atrial septal defect closure. Preliminary experience with the Hashkind occluder device. J Intervent Cardiol 1989; 2: 33-41.

7. Lock JE, Rome JJ, Davis R, et al. Transcatheter closure of atrial septal defects: experimental studies. Circulation 1989; 79: 1091-9.

8. Lock JE, Hellembrand WE, Latson L, Mullins CE, Benson L, Rome JJ. Clamshell umbrella closure of atrial septal defects: initial experience, abstracted. Circulation 1989; 80(suppl II): 592.

9. Rome JJ, Keane JF, Perry SB, Spevak PJ, Lock JE. Double umbrella closure of atrial septal defects: initial clinical applications. Circulation 1990; 82: 751-8.

10. Latson LA. Transcatheter closure of atrial septal defects. In: Rao OS, (ed). Transcatheter Therapy in Pediatric Cardiology. New York: Wiley Liss 1993: 335-48.

11. Carminati M, Giusti S, Hausdorf G, et al. A European multicentric experience using CardioSeal and Starflex double umbrella devices to close interatrial communications holes within the oval fossa. Cardiol Young 2000; 10: 519-26.

12. Pedra CA, Maclaughlin $P$, Benson LN. The role of CardioSeal and Starflex devi ces in atrial defect occlusion. Current Interv Cardiol Reports 2000; 2: 274-82.

13. Sideris EB, Sideris SE, Fowlkes JP, Ehly RL, Smith JE, Gulde RE. Transvenous atrial septal occlusion in piglets using a "Buttoned" double disc device. Circulation 1990; 81: 312-8.
14. Godart F, Rey C, Francart C, et al. Experience in one centre using the buttoned device for occlusion od atrial septal defect: comparison with the Amplatzer septal occluder. Cardiol Young 2000; 10: 527-33.

15. Rao PS, Sideris EB, Hausdorf G, et al. International experience with secundum atrial septal occlusion by the buttoned device. Am Heart J 1994; 128: 1022-5.

16. Babic UU. Double umbrella device for transvenous closure of patent ductus arteriosus and atrial septal defects. First experience. J Interv Cardiol 1991; 4: 283-94.

17. Sharafuddin MJ, Gu X, Titus JL, et al. Transvenous closure of secundum atrial septal defects: Preliminary results with a new self-expanding nitinol prosthesis in a swine model. Circulation 1997; 95: 2162-8.

18. Bjornstad PG, Masura J, Thaulow E, et al. Interventional closure of atrial septal defects with the Amplatzer device: first clinical experience. Cardiol Young 1997; 7: 277-83.

19. Wilkinson JL, Goh Th. Early clinical experience with use of the Amplatzer septal occluder device for atrial septal defect. Cardiol Young 1998; 8: 295-302.

20. Thanapoulus BD, Laskari CV, Tsaousis GS, Zarayelyan A, Vekiou A, Papadopoulos GS. Closure of atrial septal defects with the Amplatzer occlusion device: preliminary results. J Am Coll Cardiol 1998; 31: 1110-6.

21. Fontes VF, Pedra CAC, Fontes Pedra SRF, et al. Experiência inicial no fechamento percutâneo da comunicação interatrial com a prótese de Amplatzer. Arq Bras Cardiol 1998; 70: 147-53.

22. Berger F, Vogel M, Alexia Meskishvili V, Lange PE. Comparison of results and complications of surgical and Amplatzer device closure of atrial septal defects. J Thorac Cardiovasc Surg 1999; 118: 674-80.

23. Hijazi ZM, Waight DJ, Masura J, et al. Catheter closure of ASD using Amplatzer septal occluder: results of international clinical trial. AGA $3^{\text {rd }}$ International Amplatzer Symposium. Abstracts, Strasbourg, 2000: 7.

24. Latson LA, Zahn EM, Wilson N. Helex septal occluder for closure of atrial septal defects. Current Interv Cardiol Reports 2000; 2: 268-73. 